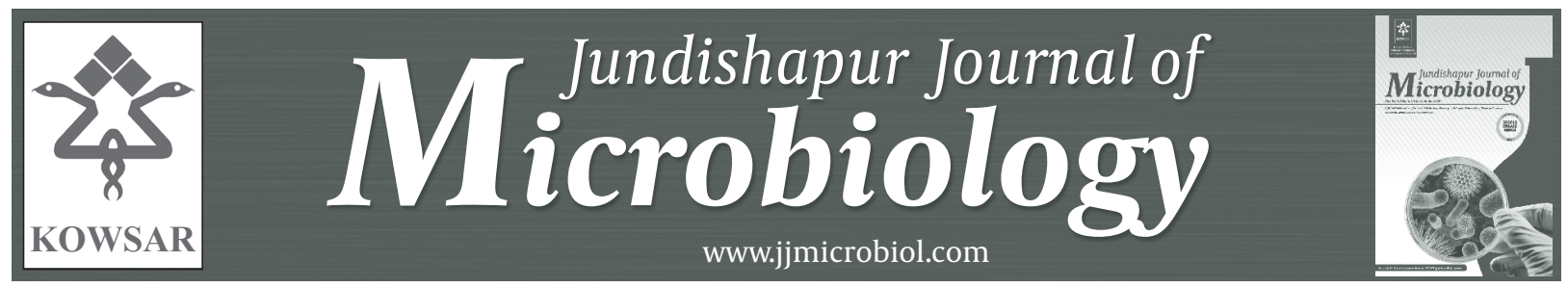

\title{
Epidemiological Surveillance of Norovirus Diarrhea in Hospitalized Children with Acute Gastroenteritis in South of Iran
}

\author{
Akram Najafi ${ }^{1,2}$, Darioush Iranpour ${ }^{3, *}$, Shariat Najafi ${ }^{2}$ \\ ${ }^{1}$ Department of Biology, Payame Noor University, IR Iran \\ ${ }^{2}$ Department of Virology, The Persian Gulf Tropical Medicine Research Center, Bushehr University of Medical Sciences, Bushehr, IR Iran \\ ${ }^{3}$ Department of Internal Medicine, Bushehr University of Medical Science, Bushehr, IR Iran \\ *Corresponding author: Darioush Iranpour, Department of Internal Medicine, Bushehr University of Medical Science, Bushehr, IR Iran. Tel: +98-9173712273, \\ Fax:+98-7712526801, E-mail: darioushiranpour@yahoo.com.
}

\begin{abstract}
A B S T R A C T
Background: Noroviruses are one of the most common causes of acute diarrhea in both developed and developing countries. They are responsible for more than $50 \%$ of all gastroenteritis outbreaks around the world.

Objectives: This study was conducted to assess the prevalence, seasonality and clinical characteristics of norovirus infection in hospitalized Iranian children.

Patients and Methods: Between 2008 and 2010, a cross-sectional descriptive study was conducted on 375 stool samples from children under 7 years of age, who suffered from acute gastroenteritis and who were admitted to the Pediatrics Unit of the 17 Shahrivar Hospital in the city of Borazjan. Acute gastroenteritis was defined as \&gt; 3 loose watery stools every 24 hours. All the stool specimens were tested for norovirus antigens with enzyme immunoassays (EIA). Demographic and clinical data were analyzed using SPSS software.

Results: Of the total collected samples, noroviruses were detected in 47 out of 375 (12.53\%). The highest infection rate was among children under two years of age $(76.6 \%)(\mathrm{P}=0.001)$. Diarrhea $(95.74 \%)$, vomiting $(87.23 \%)$ and fever $(82.98 \%)$ were the most frequently reported clinical symptoms in children with norovirus gastroenteritis. The highest prevalence of the virus was observed in autumn (63.83\%) and the lowest in summer $(6.38 \%)(\mathrm{P}=0.015)$.

Conclusions: Regarding the emergence of noroviruses as a relevant cause of acute diarrhea in Iranian children, there is a great need to introduce a routine norovirus testing of hospitalized patients with gastroenteritis, particularly in children under 2 years old during the cold season.
\end{abstract}

Keywords: Epidemiology; Norovirus; Gastroenteritis; Iran

Copyright @ 2013, Ahvaz Jundishapur University of Medical Sciences; Published by Kowsar Corp.

Article type: Research Article; Received: 23 Aug 2012; Revised: 11 Jan 2013; Accepted: 17 Jan 2013; Epub: 01 Jun 2013; Ppub: Jun 2013

Implication for health policy/practice/research/medical education:

This study improves our knowledge about the significance of noroviral gastroenteritis among hospitalized children in south of Iran.

-Please cite this paper as:

Najafi A, Iranpour D, Najafi S. Epidemiological Surveillance of Norovirus Diarrhea in Hospitalized Children with Acute Gastroenteritis in South of Iran. Jundishapur J Microbiol. 2013; 6(4).e7866. DOI: 10.5812/jjm.7866.

Copyright @ 2013, Ahvaz Jundishapur University of Medical Sciences; Published by Kowsar Corp.

This is an Open Access article distributed under the terms of the Creative Commons Attribution License (http://creativecommons.org/licenses/by/3.0), which permits unrestricted use, distribution, and reproduction in any medium, provided the original work is properly cited. 


\section{Background}

Noroviruses are a group of non-enveloped, singlestranded RNA viruses classified into the genus Norovirus of the family Caliciviridae (1). Recent studies have demonstrated that noroviruses are the second most frequent causative agents of viral diarrhea in young children after rotaviruses (2-4). A systematic review of 31 community, outpatient, and hospital-based studies estimated that noroviruses accounted for 10 to $15 \%$ of acute gastroenteritis episodes in children less than 5 years old in both developed and developing countries (5). Current estimates of annual global childhood mortality associated with norovirus infections are up to 200,000 deaths $(5,6)$.

The clinical symptoms of norovirus gastroenteritis are characterized by acute onset, non-bloody diarrhea, vomiting, nausea and abdominal cramps (1). These characteristics are the same as those observed in patients with rotavirus gastroenteritis $(7,8)$. Studies have shown that outbreaks of norovirus infections occur throughout the year, although there is a seasonal pattern of increased infection rates during the winter months (9-11). Despite the importance of norovirus infections in child morbidity and mortality rates in developing countries, very few studies have investigated the prevalence of norovirus gastroenteritis among hospitalized Iranian children with acute gastroenteritis (12-14).

\section{Objectives}

The present study aimed at assessing the frequency of norovirus infections in hospitalized children under 7 years old with acute gastroenteritis in Borazjan, Iran. In addition, we examined the age and seasonal distribution, as well as the clinical manifestations associated with norovirus in the same patient population.

\section{Patients and Methods}

From October 2008 to September 2010, a cross sectional descriptive study was conducted on 375 stool samples collected from hospitalized children under 7 years old with acute, sporadic gastroenteritis in the 17 Shahrivar Hospital in Borazjan City. Acute gastroenteritis was defined as $>3$ loose watery stools every 24 hours. All the fecal specimens were collected within 24 hours of admission. These specimens were sent to the virology laboratory of the Bushehr University of Medical Science and stored at $-70^{\circ} \mathrm{C}$ until the time of assay.

In our study, patients were removed from the described patient population if they showed symptoms of gastroenteritis with a duration of more than 7 days, antibiotic treatment before diagnosis, or confirmed bacterial diarrhea. Demographic (age and sex) and clinical data (days of hospitalization, diarrhea, vomiting, fever, convulsions, abdominal cramps and severity of dehydration) were collected for each case by using a standard structured questionnaire.

According to the WHO's recommendation, all the children with gastroenteritis were classified in specific age groups (e.g. 0-2, 3-5, 6-8, 9-11, 12-17, 18-23, months) so that age-specific incidence rates of hospitalization can be calculated (15). All the fecal specimens were tested for the presence of the norovirus antigen by using the commercially available enzyme immunoassay (EIA) kit (IDEIA ${ }^{\mathrm{TM}}$ Norovirus EIA, Oxoid, Ely, United Kingdom), according to the manufacturer's instructions.

Data was statistically analyzed using SPSS version 17 (SPSS Inc., Chicago, IL, USA). Also, $\chi 2$ test was used to analyze the data obtained for the age groups, sex and seasonal distribution of the noroviruses. Fisher's exact test was used to analyze the clinical symptoms. P value $<0.05$ was considered as statistically significant. The study was approved by the Research Ethical Committee of the Medical University of Bushehr.

\section{Results}

Noroviruses were detected in 47 (12.53\%) of all the evaluated stool samples. The distribution of gender in norovirus positive cases was $26(55.32 \%)$ in males and 21 (44.68\%) in females $(\mathrm{P}=0.08)$ (Table 1). All the patients with acute diarrhea had ages between 1 and 83 months with a median age of 22 months. Children less than 24 months of age accounted for $76.6 \%$ of the infected patients, with those children who were between 12 and 17 months of age being the most affected $(29.78 \%)(P=0.001)$.

Table 1. Distribution of Age Groups in Children With Norovirus Gastroenteritis

\begin{tabular}{|llll}
\hline $\begin{array}{l}\text { Age Groups, } \\
\text { Mo }\end{array}$ & $\begin{array}{l}\text { Norovirus } \\
\text { Group } \\
\text { 47), No. }\end{array}$ & $\begin{array}{l}\text { Non-norovi- } \\
\text { rus Group } \\
(\mathbf{n = 3 2 8}), \text { No. }\end{array}$ & $\begin{array}{l}\text { Global Num- } \\
\text { ber }(\mathbf{n}=\mathbf{3 7 5})\end{array}$ \\
\hline $\mathbf{0 - 2}$ & - & $6(1.83)$ & $6(1.6)$ \\
\hline $\mathbf{3 - 5}$ & $7(14.89)$ & $32(9.76)$ & $39(10.4)$ \\
\hline $\mathbf{6 - 8}$ & $5(10.64)$ & $40(12.19)$ & $45(12.0)$ \\
$\mathbf{9 - 1 1}$ & $9(19.15)$ & $39(11.89)$ & $48(12.8)$ \\
\hline $\mathbf{1 2 - 1 7}$ & $14(29.78)$ & $75(22.87)$ & $89(23.73)$ \\
\hline $\mathbf{1 8 - 2 3}$ & $3(6.38)$ & $37(11.28)$ & $40(10.67)$ \\
\hline $\mathbf{2 4 - 3 5}$ & $4(8.52)$ & $30(9.15)$ & $34(9.07)$ \\
\hline $\mathbf{3 6 - 4 7}$ & $2(4.25)$ & $27(8.23)$ & $29(7.73)$ \\
\hline $\mathbf{4 8 - 6 0}$ & $2(4.25)$ & $14(4.27)$ & $16(4.27)$ \\
\hline $\mathbf{6 1 - 8 3}$ & $1(2.13)$ & $28(8.54)$ & $29(7.73)$ \\
\hline
\end{tabular}


The highest prevalence of noroviruses (63.83\%) was identified in autumn, while the corresponding prevalences in winter, spring and summer were $17.02 \%, 12.77 \%$ and $6.38 \%$, respectively $(P=0.015)$. Figure 1 shows the monthly distribution of noroviruses in patients with acute diarrhea.

The survey of clinical symptoms showed that diarrhea, vomiting and fever were present in $95.74 \%, 87.23 \%$ and $82.98 \%$ of all patients with norovirus infections (Table 2). In terms of severity of dehydration, moderate dehydration with $55.32 \%$ was the most common type among children with gastroenteritis.

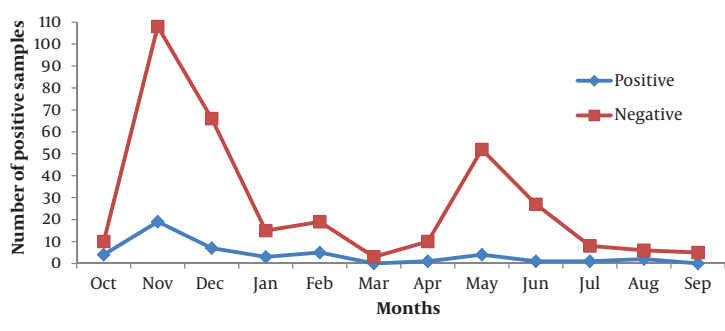

Figure 1. Monthly Distribution of Norovirus Gastroenteritis Among Children Less Than 7 Years

\begin{tabular}{|c|c|c|c|c|}
\hline Norovirus Group $(n=47)$ & Non-norovirus Group $(n=328)$ & Global Number $(n=375)$ & Pvalue & Clinical symptoms \\
\hline Diarrhea & $45(95.74)$ & $269(82.01)$ & $314(83.73)$ & 0.534 \\
\hline Vomiting & $41(87.23)$ & $139(42.38)$ & $180(48.0)$ & 0.316 \\
\hline Abdominal pain & $27(57.45)$ & $152(46.34)$ & $179(47.73)$ & 0.118 \\
\hline Convulsion & - & $10(3.05)$ & $10(2.66)$ & 0.427 \\
\hline Fever & $39(82.98)$ & $86(26.22)$ & $125(33.33)$ & 0.04 \\
\hline \multicolumn{5}{|l|}{ Dehydration severity } \\
\hline Severe & $15(31.91)$ & $47(14.33)$ & $62(16.53)$ & 0.153 \\
\hline Moderate & $26(55.32)$ & $173(52.74)$ & $199(53.07)$ & 0.728 \\
\hline Mild & $6(12.77)$ & $108(32.93)$ & $114(30.4)$ & 0.06 \\
\hline
\end{tabular}

\section{Discussion}

Despite considerable advances in current public health services and hygiene control, diarrhea still remains a potential risk to human health, especially in infants and young children worldwide (16). Recently, many studies have demonstrated the importance of noroviruses as one of the most common leading causes of viral epidemic diarrhea in both developed and developing countries (1719). In our study, the incidence of noroviruses (12.53\%) is in agreement with those reported in previous studies in Iran $(13,14)$ and in other countries $(10,17,18)$.

The occurrence of norovirus infections that were observed in the first 24 months of life (76.6\%) is greater than in the older age groups, as also found in studies conducted in Spain (10), Taiwan (11) and Vietnam (20). The high frequency of norovirus in this age group indicates that children under 2 years of age, are at greatest risk for developing acute gastroenteritis from norovirus. Norovirus positive cases were detected in males more often than in females, as was found in other investigations $(9,21)$.

In the present study, norovirus-induced gastroenteritis episodes were observed during the whole year, with the exception of March and September. We also observed a peak wave from November through December. These results are consistent with previous studies that have identified the highest prevalence of norovirus infection during the cold months of the year $(11,13,19,21)$. In the current study, most commonly reported symptoms were diarrhea, vomiting and fever. These findings are in accordance with those studies conducted in Iran (14), Spain (10), Hungary (9) and Venezuela (22).

Because of some limitations in sensitivity and specificity, EIA cannot be a considered as a reliable method for the detection of the infectious pathogens in stool samples. In recent years, molecular methods have rapidly found their way into virological studies. Generally, different studies have considered the PCRs as specific and sensitive techniques used to detect enteric viruses, such as norovirus and rotavirus, in samples (23). For this reason, we suggest using PCRs methods to assess the presence of noroviruses in upcoming surveillance studies.

One limitation of the current study is that the true frequency of norovirus infections could be higher than estimated in the present study (12.53\%), because only hospitalized children with moderate to severe gastroenteritis were included, and the proportion of norovirus infections in children with only home care or outpatient visits has not been evaluated. Another possible limitation is that the results in this study may not be representative for all 0 to 7-year-old Iranian children. Since we identified the prevalence of norovirus-induced gastroenteritis 
from only one city in Iran, in order to have a comprehensive picture of the disease burden of noroviruses in this country, it is necessary to conduct the same studies in other regions of Iran.

In conclusion, our results provide information on the epidemiology and the prevalence of noroviruses in children with acute gastroenteritis in Borazjan, a city in the south of Iran. We conclude that noroviruses are frequent causes of acute sporadic gastroenteritis, requiring the hospitalization of Iranian children under 7 years of age. It affects mainly children who are less than 2 years old and is more predominant during the cold seasons. Regarding the emergence of noroviruses as a relevant cause of acute diarrhea in Iranian children, there is a great need to introduce routine norovirus testing among hospitalized children with gastroenteritis in order to recognize the real disease burden of viral intestinal infections in the Iranian child population.

\section{Acknowledgements}

The authors wish to extend their thanks and appreciation to Dr. Hassan Shafiei for his executive support of this project. We also would like to thank the staff of the 17 Shahrivar Hospital in Borazjan who assisted us in performing this project and collecting samples.

\section{Authors' Contribution}

None declared.

\section{Financial Disclosure}

None declared.

\section{Funding/Support}

This study was supported in part by grant 4895B from Payame Noor University, IR Iran.

\section{References}

1. Updated norovirus outbreak management and disease prevention guidelines. MMWR Recomm Rep. 2011;60(RR-3):1-18.

2. Oldak E, Sulik A, Rozkiewicz D, Liwoch-Nienartowicz N. Norovirus infections in children under 5 years of age hospitalized due to the acute viral gastroenteritis in northeastern Poland. Eur $J$ Clin Microbiol Infect Dis. 2012;31(4):417-22.

3. Tran A, Talmud D, Lejeune B, Jovenin N, Renois F, Payan C, et al. Prevalence of rotavirus, adenovirus, norovirus, and astrovirus infections and coinfections among hospitalized children in northern France. J Clin Microbiol. 2010;48(5):1943-6.

4. Zhang S, Chen TH, Wang J, Dong C, Pan J, Moe C, et al. Symptomatic and asymptomatic infections of rotavirus, norovirus, and adenovirus among hospitalized children in Xi'an, China. J Med Virol. 2011;83(8):1476-84.

5. Patel MM, Widdowson MA, Glass RI, Akazawa K, Vinje J, Parashar UD. Systematic literature review of role of noroviruses in sporadic gastroenteritis. Emerg Infect Dis. 2008;14(8):1224-31.
6. Kaplan NM, Kirby A, Abd-Eldayem SA, Dove W, Nakagomi T, Nakagomi $\mathrm{O}$, et al. Detection and molecular characterisation of rotavirus and norovirus infections in Jordanian children with acute gastroenteritis. Arch Virol. 2011;156(8):1477-80.

7. Huh JW, Kim WH, Yoon MH, Lim YH. Genotypic distribution of rotavirus strains causing severe gastroenteritis in Gyeonggi province, South Korea, from 2003 to 2005. Arch Virol. 2009;154(1):16770.

8. Najafi A, Shafiei H, Barazesh A, Najafi S, Mohammadian MK, Vahdat K, et al. Epidemiological and clinical characteristics of gastroenteritis associated with enteric adenovirus in hospitalized children in Bushehr Province, Iran. African J Microbiol Res. 2011;5(30):5402-5406.

9. Jakab F, Nemeth V, Oldal M, Varga L, Nyul Z, Mitchell DK, et al. Epidemiological and clinical characterization of norovirus infections among hospitalized children in Baranya County, Hungary. J Clin Virol. 2010;49(1):75-6.

10. Junquera CG, de Baranda CS, Mialdea OG, Serrano EB, SanchezFauquier A. Prevalence and clinical characteristics of norovirus gastroenteritis among hospitalized children in Spain. Pediatr Infect Dis J. 2009;28(7):604-7.

11. Yang SY, Hwang KP, Wu FT, Wu HS, Hsiung CA, Chang WC, et al Epidemiology and clinical peculiarities of norovirus and rotavirus infection in hospitalized young children with acute diarrhea in Taiwan, 2009. J Microbiol Immunol Infect. 2010;43(6):506-14.

12. Adabian Saadat, Fallah Fatemeh, Gachkar Latif, Jadali Farzaneh, Tabatabaei S Rafiei, Esmaeilnejad Narges. Detection of norovirus in stool samples by RT-PCR in 5 disease centers in Iran. BMC Infect Dis. 2012;12(Suppl 1):O10.

13. Jalilian S, Samarbaf-Zadeh A, Mozhgani S, Makvandi M, Parsa-nahad M, Pirmoradi R, et al. Relative frequency of norovirus infection in children suffering from gastroenteritis referred to Aboozar hospital, Ahvaz, Iran.Jundishapur JMicrobiol. 2012;5(1):355-358.

14. Romani S, Mohebbi SR, Hosseini SM, Azimzadeh P, Vahedi M, Derakhshan F, et al. Prevalence of norovirus infection in children and adults with acute gastroenteritis, Tehran, Iran, 2008-2009. Food Environ Virol. 2012;4(1):1-5.

15. Generic protocols for (i) hospital-based surveillance to estimate the burden of rotavirus gastroenteritis in children and (ii) a communitybased survey on utilization of health care services for gastroenteritis in children. 2002

16. Black Robert E, Cousens Simon, Johnson Hope L, Lawn Joy E, Rudan Igor, Bassani Diego G, et al. Global, regional, and national causes of child mortality in 2008: a systematic analysis. Lancet. 2010;375(9730):1969-1987

17. Colomba C, Saporito L, Giammanco GM, De Grazia S, Ramirez S Arista S, et al. Norovirus and gastroenteritis in hospitalized children, Italy. Emerg Infect Dis. 2007;13(9):1389-91.

18. Rasanen S, Lappalainen S, Salminen M, Huhti L, Vesikari T. Noroviruses in children seen in a hospital for acute gastroenteritis in Finland. Eur J Pediatr. 2011;170(11):1413-8.

19. Zeng M, Gong Z, Zhang Y, Zhu Q, Wang X. Prevalence and genetic diversity of norovirus in outpatient children with acute diarrhea in Shanghai, China.Jpn J Infect Dis. 2011;64(5):417-22.

20. Tamura T, Nishikawa M, Anh DD, Suzuki H. Molecular epidemiological study of rotavirus and norovirus infections among children with acute gastroenteritis in Nha Trang, Vietnam, December 2005-June 2006. Jpn J Infect Dis. 2010;63(6):405-11.

21. Fretz R, Herrmann L, Christen A, Svoboda P, Dubuis O, Viollier EH et al. Frequency of Norovirus in stool samples from patients with gastrointestinal symptoms in Switzerland. Eur J Clin Microbiol Infect Dis. 2005;24(3):214-6.

22. Gonzalez GG, Liprandi F, Ludert JE. Molecular epidemiology of enteric viruses in children with sporadic gastroenteritis in Valencia, Venezuela. J Med Virol. 2011;83(11):1972-82.

23. Girones R, Ferrus MA, Alonso JL, Rodriguez-Manzano J, Calgua B, Correa Ade A, et al. Molecular detection of pathogens in water--the pros and cons of molecular techniques. Water Res. 2010;44(15):4325-39. 